\title{
El castillo de Rochafrida entre la literatura y la historia
}

\author{
Angela MADRID y Medina *
}

1. Sorprende encontrar en el corazón de Castilla a héroes de Roncesvalles. Aunque sea de la mano del más estrafalario de los caballeros andantes ${ }^{1}$. Pero choca aún más la descripción de las Relaciones Topográficas ordenadas por Felipe II, que no hay razón para pensar que estén concebidas por mentes tan descarriadas ${ }^{2}$.

Es curioso que los romances, cuyo carácter noticiero no hay que maximalizar, con mayor razón si son tardíos, se afiancen con tal terquedad a unos relatos y a unos personajes. Entendemos, con todo, que don Quijote recoja la ambientación caballeresca del romancero, dentro de su

* Prof. tutora UNED-Valdepeñas.

Utilizo la edición del Quijote de Plaza y Janés, S.A., 1980, con ilustraciones de Gregorio Prieto, págs. 169-86.

${ }_{2}$ «A la parte de levante en una laguna que se dice que no tiene mucha agua y que en agosto se suele apocar y enjugar y que no quedan sino aguachares hay una fortaleza en medio de la dicha arruinada, el edificio della que comunmente se llama en esta tierra el castillo de Rochafrida donde dicen que antiguamente estuvo una doncella que llamaron Rosaflorida muy hermosa y siendo señora de aquel castillo la demandaron en casamiento Duques y Condes de Lombardía y otras partes estrañas y a todos los despreció e oyendo decir nuevas de Montesinos se enamoró del y lo envio buscar... y se caso con el que era hombre de notable estatura... y que en aquel castillo vivieron juntos... y cerca... esta una cueba... de Montesinos... esta el dicho heredamiento y lo demas en termino de Alhambra termino comun a la dicha villa de la Solana y a las otras de la Orden de Santiagon. C. VINAS y R. PAZ: Relaciones de los pueblos de España ordenadas por Felipe II. Ciudad Real, Madrid, CSIC, 1971. La Solana, pág. 481 y 482. 
desvarío ${ }^{3}$. Nos cuesta, sin embargo, explicar los motivos del informante de las "Relaciones" al plasmar de forma tan fidedigna el romance de Rosaflorida ${ }^{4}$.

Pero, despojando los textos del lenguaje literario, prescindiendo de los siete condes y los tres duques, de si Rosaflorida tiene «mal de amores» o está "loca sandía» ${ }^{5}$ y del, según Di Stefano, "desamor apacible» de Durandarte ${ }^{6}$, nos queda el castillo de Rochafrida, en el Campo de Montiel, a una legua del nacimiento del río Guadiana y que, a decir de Corchado, que se apoya, con fundamento, en su ubicación, no es otro que el más antiguo de San Felices ${ }^{7}$ donado por Enrique I a Suero Téllez de Meneses.

2. Suero Téllez y su familia son gentes de frontera. La crónica de Calatrava nos lo menciona entre los caballeros castellanos que intervinieron en la batalla de las Navas de Tolosa ${ }^{8}$. Es, sin embargo, Julio González ${ }^{9}$ quien nos sitúa al personaje. Entre los descendientes de Pedro Ansúrez, fundador de Valladolid.

Fue don Suero hijo de Tello Pérez, quien desde 1168 hasta 1193 aparece de manera más o menos ininterrumpida confirmando privilegios reales. A él y a Pedro Gutiérrez les otorgaría Alfonso VIII el castillo y la villa de Ocaña el 11 de abril de 1173. A don Tello y su mujer, doña

3 "Oh Belerma, oh Belerma/por mi mal fuiste engendrada, que siete años te serví/sin de ti aicanzar nada;/agora que me querías, muero yo en esta batalla/... Oh mi primo Montesinos, /lo que agora yo os rogara/que cuando yo fuera muerto/...vos lleveis mi corazón/ adonde Belerma estava". El Fomancero, con un estudio de G. DI StEFAno. Madrid, ed. Narcea, S.A., págs, 193-95.

4 «En Castilla está un castilio,/que se llama Rocafrida/...dentro estaba una doncella/ que llaman Rosaflorida:/siete condes la demandan,/tres duques de Lombardía;/a todos los desdeñaba,/tanta era su lozanía./Enamorose de Montesinos/de oidas, que no de vista/...". El Romancero. Introducción y selección de M. ALVAR, Madrid, ed. S.M., 1974, págs. 190191. En la recopilación de Di Stefano, págs. 153 y 154.

5 Ibidem.

6 Op. cit. págs. 50 y 146-147. Me refiero al romance que comienza «Durandarte, Durandarte».

7. M. Corchado Soriano: Avance de un estudio geográfico-histórico del Campo de Montiel, I.E.M., 1971, págs. 142 y 143.

a F. de RADES y ANDAADA: Crónica de las tres Ordenes de Santiago, Calatrava y Alcántara, Barcelona, «El Albir», S.A., 1980. Edición facsimil, f. ${ }^{\circ} 27$ v. ${ }^{\circ}$.

¿ En El Reino de Castilla en la época de Alfonso VIII, Madrid 1960, t. II, págs. 34752. 
Gontrodo, les dará el 10 de noviembre del mismo año el infantazgo de Matallana - con anterioridad, 3 de enero, les había entregado la mitad de Ocaña, junto con varias villas y heredades a cambio de Malagón-. Con otra serie de privilegios. En 1181 los escribanos denominan a don Tello señor de Meneses y lo consideran «familiarius regis», siguiendo a J. González. Interviene activamente en la zona de Campos y de Cea. Al margen de otros privilegios, detallados por el mencionado medievalista, nos importa señalar la predilección de Tello Pérez por las Ordenes Militares y por las fundaciones hospitalarias: el 6 de diciembre de 1196 funda el de Villa Martín, en el camino de Santiago, que confió a la Orden de igual nombre para atender a siete leprosos. El mismo destino tiene el de San Nicolás del Camino, también a instancias suyas. Y más al sur regala, en unión de su mujer y de Pedro Gutiérrez y la suya (María Bueso) una heredad para el hospital de cautivos de Cuenca ${ }^{10}$.

A su sucesor, Alfonso Téllez, lo encontramos, además, en la Crónica de Santiago ${ }^{11}$. Casado sucesivamente con Elvira Rodríguez y con Teresa Sánchez, hubo del primer matrimonio a Tello, Alfonso y Mayor y del segundo probablemente, según González, a Teresa Alfonso ${ }^{12}$. Era Teresa Sánchez, dice la Crónica, hija del rey Sancho de Portugal y aportó al matrimonio el castilio de Alburquerque. Según dicha fuente, que afirma que la primera mujer fue Elvira Girón, con doña Teresa tuvo a Juan Alonso, Alonso Téllez Tizón y Martín Tello. Y añade que los dos Alonso Téllez (de ambos matrimonios) «hizieron Pleyto omenage de ayudarse el uno al otro contra todos los hombres del mundo: fueras ende su señor y sus hermanos y sus primos cormanos don Gutierre y don Suero, hijos de don Suer Tellez» ${ }^{13}$. Por su parte Tello Alfonso y Alonso Téllez manifiestan su preocupación por la Orden de Santiago favoreciéndola con diversos heredamientos en Ballesteros para el hospital de Toledo ${ }^{14}$.

Y será probablemente Alfonso Téllez, hijo, el personaje que encontramos ya documentado en el Campo de Montiel. El 15 de marzo de 1227 confirmando el privilegio dado en Carrión por Fernando III por el que concede al maestre Pedro González y a la Orden de Santiago los

10 También favorecido por el rey Alfonso y su mujer doña Leonor, según una referencia muy posterior, del año 1478. AHM Orden Militar de Santiago. Libros manuscritos. Visitas, $1063 \mathrm{C}$, f. $^{\circ} 199$.

11 RADES, Op. cit. F. 29 v. ${ }^{\circ}$ y 30.

12 González, Op. cit., pág. 351.

13 Rades. Op. cit., f. 30.

14 lbidem, F. 29 v. 
castillos de San Polo y la Estrella de Montiel con todas sus pertenencias ${ }^{15}$. En Valladolid, 18 de febrero de 1242 confirma otro privilegio del mencionado monarca para que le sean devueltos a los caballeros de Santiago los términos que su abuelo don Alfonso les había entregado y ellos habían repoblado. Saliendo al paso de un pleito con Alcaraz. Se cita, entre otros lugares, Ruidera ${ }^{16}$. El mismo año, 11 de septiembre, en Burgos, confirma uno más por el que San Fernando concede a la Orden las iglesias de Villanueva ${ }^{17}$.

Durante el reinado de Alfonso $X$ y con respecto al Campo de Montiel encontramos el nombre de Alfonso Téllez al menos en dos confirmaciones. La de la carta plomada de 8 de agosto de 1252, Sevilla, por la que el rey concede al maestre Pelay Pérez Correa, por la ayuda que le presta en la conquista de Murcia, que se celebre una feria en Montiel ${ }^{18}$. Y en un privilegio real de 5 de julio de 1256, mediante el cual, el monarca otorga al comendador de Segura, el cortijo de Abeiazat «por el servicio que me fizo sobre Oriuela quando la gane» ${ }^{19}$.

El mismo Suero Téllez, hijo de don Tello Pérez, aparte de en otras confirmaciones citadas por J. González ${ }^{20}$ y Milagros Rivera ${ }^{21}$ y las referencias ya indicadas, de la crónica santiaguista, aparece documentado pronto en el Campo de Montiel. Confirma un privilegio dado en Burgos por Alfonso VIII, el 3 de mayo de 1214, por el que concede, por razones espirituales, al maestre García González el castillo de Eznavejor, fortaleza ganada a «paganos» en el «tercio uidelicet anno postquam ego dictus rex Adefonsus Almiramo-meninus reges de Marrocos apud Nauas de Tolosa campesti proelio superaui non meis meritis sed Dei clementia et meorum auxilio uassallorum ${ }^{22}$.

3. No es frecuente que el rey entregue a un noble, un castillo del Campo de Montiel para su defensa y la repoblación del territorio. Lo

\footnotetext{
AHN OO.MM. Carp. 214, n. ${ }^{\circ}$.

Ibidem. Carp. $365, n .^{\circ} 2$.

Ibidem. Carp. 365, n. ${ }^{\circ} 4$.

18 Ibidem. Carp. 214, $n .{ }^{\circ} 11$. Aparte de alguna cita anterior, la reproduzco integramente en mi pequeña aportación, que fue, a la vez, mi primer acercamiento a la Historia Medieval, en el Congreso Internacional de Alfonso X, celebrado en 1984.

${ }^{19}$ Ibidem. Caxón $315, n .^{\circ}$. También está reproducido en su integridad en mi comunicación al citado Congreso.

${ }^{20}$ Op. cit., págs. 351 y 352.

21 M. Rivera Garreta: La Encomienda, el Priorato y la Villa de Uclés en la Edad Media, Barcelona, CSIC, 1985.

${ }_{22}$ AHN DO. MM. Carp. 214, n. 2.
} 
habitual es que lo haga a la Orden de Santiago. Pero el caso de Suero Téllez y San Felices tampoco es único. El mismo Enrique I donó, en precario, el castillo de Alhambra en 1217 al Conde Alvaro Núñez de Lara ${ }^{23}$.

Del anacoreta del siglo v San Felices (o San Félix) Gonzalo de Berceo nos transmite información en la vida de San Millán ${ }^{24}$. Murió el Santo en el castillo de Bilibio, siendo sepultado en el oratorio del mismo ${ }^{25}$. En 1090 su cuerpo se trasladó al monasterio de San Millán, lugar en el que permanecen sus reliquias junto a las del santo titular. Bilibio por su parte pasó a denominarse San Felices. Y en la actualidad San Félix es el patrono de Haro ${ }^{26}$. No es de extrañar que en los territorios recién conquistados se utilice el nombre de santos de devoción tan manifiesta.

Como indicábamos antes, el Castillo de San Felices, en la Mancha santiaguista, fue donado a don Suero Téllez de Meneses, que tan amplia vinculación tenía con la Orden. Enrique I se lo entrega «pro multis et gratis seruiciis» con todas sus pertenencias y con plena libertad. Además "dono itaque nobis et concedo hereditatem illam que es in término de Alcáraz que Ossa dicitur». El documento, sin año, está datado en Castrogeriz, el 26 de abril. Entre los confirmantes volvemos a encontrar a Alfonso Téllez ${ }^{27}$. El privilegio es confirmado sucesivamente por Fernando III, en 1222, y por su hijo, el rey Alfonso, en 1255, que internacionaliza el documento con la cita de Eduardo de Inglaterra y la confirmación de los emperadores de Constantinopla y de sus hijos ${ }^{28}$.

Tras la donación nos encontramos con el proceso de delimitar los términos de San Felices. En dos documentos casi coetáneos. El primero

23 Lo cita J. González, M. Corchado, el Bulario de las Ordenes Militares y yo misma. Puede verse, además, el texto íntegro de un vidimus del mismo, procedente del archivo municipal de Alhambra, sin catalogar, en mi artículo «Repoblación Manchega Bajomedieval: la Carta Puebla de Villamanrique", Cuadernos del IEM, n. ${ }^{\circ} 16,1985$.

24 «Mientre iaçie dormiendo fue de Dios aspirado./ Quando abrió los ojos despertó maestrado./ Por partirse del mundo oblidó el ganado./ Entendió que el mundo era pleno de enganno/ Querie partirse delli, ferse ermitanno/...Sopo que Sant Feliçes en Bilibo moraba/ ...Entró en el castiello, falló al castellero./ Al varon benedito, al feliz caballero./ Al ermitan orando en somo del otero./ Plus umildosa-mientre que un monge claustrero». En Obras Completas, Logroño, Instituto de Estudios Riojanos, 1981, págs. 126-27.

25 P. J. Croisset: Año Cristiano, t. II, Madrid, Imprenta Gaspar y Roig, 1852, pág. 580.

J. Cantera Orive: Santos de la Rioja, Logroño. Museo Catequístico Diocesano, págs. 24-28. Mi agradecimiento a don José Repetto por su orientación bibliográfica.

${ }^{27}$ AHN Secc. Clero. Carp. 214, n. ${ }^{\circ} 3$.

28 Apéndice, III. 
es una carta de compromiso entre Gutierre Suárez, hijo de Suero Téllez, y el maestro Pelay Pérez Correa. Una carta, partida en ABC fechada el 4 de abril de 1254. Los mojones se sitúan a una legua, tomando tierras de Alhambra, Ruidera y del resto de los territorios de la Orden. Entre los testigos Juan Alfonso, hijo de Alfonso Téllez ${ }^{29}$.

En el segundo, el 23 de junio del mismo año, Suero Téllez, hijo de Suero Téllez de Meneses y hermano de Gutierre Suárez, procede a la partición del término de San Felices con hombres buenos convocados al efecto ${ }^{30}$.

La Orden de Santiago, en un proceso de implantación en la Mancha, y de consolidación del territorio, acabará intercambiando diversos lugares de Valladolid y Palencia que Suero Téllez, hijo, y su mujer Aldonza Fernández le habían legado, con Gutierre Suárez y Elvira Yañez, su mujer, quienes entregan a Pelay Pérez Correa la Ossa y dos Barrios ${ }^{31}$. Y es importante, porque, como afirma J. González ${ }^{32}$, a partir de aquí la Orden de Santiago se dedicó a repoblar Socuéllamos --tomando como base el cortijo de Abeiazat ya mencionado-, Campo de Criptana y Pedro Muñoz.

Lo demás se diluye y queda tras el velo literario. Que la ermita, próxima al castillo y que todavía estaba en pie en el primer tercio de este siglo, de San Pedro de Sahelices sea fundada por Montesinos, que acaso fuese prisión de santiaguistas ${ }^{33}$ o que tratemos de bucear en la memoria popular en busca de una referencia. $Y$ más incognitas. Sabemos que en 1478, la iglesia de La Solana aún estaba en construcción ${ }^{34}$, aunque Corchado apunta que se levanto en el siglo XVI junto a la torre de la defensa ${ }^{35}$. Pero, mientras que en la Visita de la mencionada fecha, la fortaleza de Alhambra se minimiza, destacan en esa encomienda la torre y cortijo de La Solana, si bien añadiendo que estaba en la villa. Hacen referencia al ancho muro de la torre y a unas puertas de madera forradas de cuero con cerraduras y tranca. Del cortijo dicen que es de tres tapias en alto, que las puertas del postigo se encuentran en un portal

29 Apéndice, 1.

Apéndice, II.

Apéndice, IV.

2 En VII Centenario del Infante don Fernando de la Cerda, IEM, 1976, pág. 19.

CoRChado, Op. cit., pág. 152.

34 Puede verse mi comunicación al Congreso de Devoción Mariana y Sociedad Medieval, celebrado en Ciudad Real en marzo de 1988. En vía de publicación.

35 CoRchado, Op. cit., pág. 162. 
con un arco. Hablan de la cocina con chimenea, de la despensa, del establo y de un atajo que, desde el cortijo, va hasta el muro de la torre. Citan un palacio con establo para doce bestias y dos jaraices. Mosén Diego de Villegas, padre del comendador, Diego Rodríguez de Villagarcía, había realizado varias obras en la torre. Los visitadores mandan reparar los tejados. Por lo que se refiere a armamento, consistía éste en cuatro ballestas de acero, seis de palo fuerte con dos tornos y una garrucha, siete espingardas, un trueno gastado, pólvora, una arroba de pelotas y diez docenas de pasadores. Dicen los visitadores que hay que añadir seis paueses y seis lanzas ${ }^{36}$.

¿Por qué la Visita no relaciona la torre y el cortijo con la iglesia? ¿Por qué al hablar de Ruidera ${ }^{37}$ no existe ninguna alusión al castillo de San Felices o de Rochafrida, cuando, sin embargo, lo citan, como hemos visto, en las posteriores Relaciones Topográficas? No es nuestra intención plantear hipótesis que, desde el punto de vista científico carecerian de base sólida.

Sea como fuere ocurre que a veces, Literatura e Historia confluyen. $Y$ ahora lo han hecho en un enclave santiaguista de La Mancha, inmerso en el proceso repoblador del siglo XIII, con personajes que, como Suero Téllez y su familia - y el propio maestre Pérez Correa - nada tendrían que envidiar a los míticos Montesinos y Durandarte.

${ }^{36}$ Libro de visitas citado, $F^{\circ} 251$ y 252.

${ }^{37}$ Ibidem, f. ${ }^{\circ} 215$. 



\title{
APÉNDICE DOCUMENTAL
}

\author{
DOCUMENTO I
}

1254, abril, 4

Carta de compromiso entre la Orden de Santiago de una parte y Gutierre Suárez y su hijo de otra, sobre participación y amojonamiento de San Felices.

AHN Ordenes Militares. Carp. 214, n. ${ }^{\circ} 12$.

In dei nomine. Connosçida cosa sea a los todos los omes que esta carta vieren tanbien a los que son cuemo a los que ha por seer cuemo yo don Pelay Pedrez por la gracia de Dios Maestre de la caualleria de Sant Yago e yo don Gutier Suarez hijo de don Suer Tellez ponemos tal postura sobre los terminos de Sant Felizes que yo don Gutier Suarez e de partir con la orden e con el maestre don Pelay perez es qontra lo que Alfanbra y es qontra la Roydera e contra todas las partes de la orden. $E$ yo don Pelay Perez el Maestre sobredicho e yo don Gutier dimos e otorgamos por partidores a don Gutier Gonzaluez Quexada, amos a dos o el uno dellos qualquier que y pueda ser. $E$ deuen dar una legua de castiello de Sant Felices a todas partes es quntra la heredad de la orden e poner los moiones en cabo de la legua, et este pleito deue seer conplido fasta esta fiesta primera de la cinquagesima que viene en el era desta carta. E por do estos dos partidores sobredichos partieren e pusieren los terminos segund cumplamos e que lo ayamos por firme nos y el cabildo para siempre iamas e fazemos en esta carta poner nuestros seellos. E yo don Gutier Suarez otrosi los otorgo de la mi parte e pongo y 
el mio seello. E yo el maestre sobredicho do por fiador para complir tod esto sobredicho a don Alfonso Tellez en dos mil maravedis. $E$ yo don Alfonso Tellez despues que oy esta carta e la entendi, otorgo sobre mi e sobre todo quanto que e muebles e rayz que cumpla al maestro todo quanto en esta carta se dize. $\mathrm{E}$ el que este pleyto non quisiere complir que este fiador de a la otra parte dos mil maravedis y el pleyto con la partición e con los moiones sea todauia estable por siempre iamas. E yo don Alfonso Tellez otrosi lo otrogo. E pedimos merced a nuestro sennor el Rey don Alfonso que este pleyto que lo faga todo complir si alguna de las partes quisiere fallecer. $E$ que este sea mas firme e mas estable e non venga en dubda mandamos fazer dos cartas partidas por A.B.C. E yo el maestre sobredicho mando poner mio seello pendiente y a este cabildo general primero que ha de seer fare y poner el seello del cabildo otrosi pendiente, e si el cabildo non y pusiere su seello cuemo dicho es que el fiador sobredicho sea servido de dar los dos mil maravedis a don Gutierre el sobredicho. E yo don Alfonso Tellez el fiador connombrado assi lo otorgo. $E$ yo don Gutier Suarez pongo luego y el mio seello. Presente que este pleyto uieron e oyeron. Don Johan Alfonso so fijo de don Alfonso Tellez. Don Martin Alfonso so hermano, don Pedro Gonçaluez Quexada, Suer Garcia fijo de don Garci Suarez, Roy Hernandez, Roy Gonçalvez, Estevan Domingo Davidal, Munio Royz de Villanueva de Xamanço. Gonçaluo Garcia de Consolación, Gonçaluo Perez, Gonçaluo Gutierrez de Villa calabuey, Pedro Martinez de Toledo, Aluar Negro, Gonçaluo Royz, Aluar Garcia, Pedro Garcia de Villa Grada, Roy Martinez de Cea. Los Freyres que fueron presentes e que uieron esto e lo oyeron, Don Pedro Diaz, Don Pedro Mafalda, Don Melend Yuañez, don Gomez Pelaez, Ferrand Yvañez capellan del maestre, Pedrivañez, don Lope Rodriguez, don Diago Muñoz. Blasco Perez mayordomo del maestre. Don Domingo capellan de Toledo, don Frey Ihuan. E yo don Pelay Pedrez el maestre sobredicho e yo don Gutier Suarez el connombrado e yo don Alfonso Tellez el fiador de suso dicho pues que oyemos estas cartas que mandamos fazer e las entendimos otorgamos quanto escripto es en ellas ante las firmas que escreuiran sus nombres en finamiento dellas. Fecha la carta en quatro dias del mes de Abril. En era de mill e dozientos e nonaenta e dos annos. 
DOCUMENTO ॥

1254, junio, 23

Compromiso entre Pelayo Pérez Correa y Gutierre Suárez mediante el cual Suerro Téllez y Gutierre González Quesada cortan para el castillo de San felices una legua de término a costa de la Orden.

AHN Ordenes Militares. Carp. 214, n. ${ }^{\circ} 3$.

Conocida cosa sea a todos los omes que esta carta uieren como yo Suer Tellez sobre abenencia que hiço don Pelay Pedrez Maestre de la caualleria de San Jague con don Gutier Suarez que partieremos yo e Gutier Gonzales Quexaca al termino en que San Felices et la orden de Ucles e que tomariemos del castiello de Sant Felices una legua a todas partes contra termino de la orden de Ucles asi como dizen las cartas de la abenençia que ficieron que son partidas por abec. e selladas con sus sellos e si por auentura yo Suer Tellez e Gutier Gonzalez non pudieramos abisados uenir a partir el termino que lo partiese qualquier de nos que uiniese e uin yo don Suer Tellez e tome omes benos. Garcia Fernandez freyre del Ospital e comendador de Arenas e de Erencia e fue con el don Pedro de Cruz Fumos e Pedro de Fernandez morador en Santa Maria Guadiana e don Pedro Yllan maestre de Alcaraz e don Miguel clerigo de Alcaraz e Johan de Robres e Domingo Alcaniz moradores en Peniella. Diego Lopez e Domingo Pascual moradores en Sotouedamos e Garci Royz de Moral de la Rana e Goncaluo Martinez de Medina e Pedro Garcia de Villa egral e Ferrant Ferrandez de Camora e partillo con estos omes sobredicho. E pus el primero moyon en la Peña ruuia o esta el moyon de los de Alcaraz con los de Montiel e con don Gutierre et la Cañada Ayuso por somo de las Campoñones et por somo de la Laguna Blanca como va derecha a la carrera e va del Capiello a la 
Rruydera e de la Laguna Blanca fasta la carrera posterior III moyones e el uno esta en somo de una ençica que esta alonde de la carrera e desde ayuso es la carrera moyon fasta a la Fuente del Mando et de la Fuente del mando derechos al castillo de la Muela e dali al cabo del Ayaçira o es la Torre que lauro Goncaluo Royz. E parada de la Aljalcira la Cañada de suso (ilegible) fasta el Villar de la Manga et deste moyon como va derecho al porteçuello del Poço del Caualgador cara al moyon de los de Alcaraz con los de el Ospital e con los freyres de Ucles e con don Gutierrez e porque este fecho desta particion sea conoçida e non uenga en dubda mande yo Suer Tellez facer III cartas partidas por abec selladas con mis sellos e di la una a don Pelay Perez Maestre de la caualleria de Sant Jague e la otra a don Gutier Suarez e la otra tengo yo Suer Tellez. Fecha la carta XXIII dias de Junio era mil e CC e Nouenta e dos años. 
DOCUMENTO III

1255, septiembre, 3. Valladolid

Privilegio real de Alfonso $X$ confirmando otro de su padre en que concede la Osa y San Felices a Suero Tellez.

AHN OO.MM. Sec. diplomática. Carp. 214, n. 15.

(Christus, alfa y omega). Connoscuda cosa sea a todos los ommes que esta carta vieren cuemo yo don Alfonso por la gracia de Dios rey de Castilla de Toledo de Leon de Gallicia de Seuilla de Cordoua de Murcia e de Jahen vi un priuilegio del Rey don Fernando mio padre fecho con esta guisa. Decet reges ac principes catholicos contra insidas infidelium fines eorum seu frontaria premunire ne cum inpossessiones fidelium ipsi uiuerint munici catholicorum negligencia aminitant filoque tam modernis quam posteris uolo fieri manifestum quod Fernando Dei gratia rex Castelle et Toleti una cum uxore mea regina donna Beatrice et filio meo Alfonso ex assensu et beneplacito itaque uobis concedo et confirmo hereditatem illam que Ossa dicitur in frontaria maurorum in confinibus Alcaraz et Sanctum Felicem cum pascuis nemoribus deffesis aquis riuis fontibus pratis et cum terminis illis cum quibus illam nobis dedit auunculus meus rex Enricus predecessor meus et omnibus perteneciis suis ut illam propeletis et habeatis liber et irreuocabiliter iure hereditario in perpetuum ad fastiendum inde quidquid uolueritis dando uedendo comcambiando impignorando seu quod libet aliud iuxta uestrum libitum fatiendo. Siquis uero hanc cartam infringere seu diminuere in aliquo presumpserit iram Dei omnipotentis plenarie incurrat et cum luda Dominum perditore penas sustineat infernales et regie parti mille aureos in coto persoluat et damnum uobis hiper hoc illatum restituat duplicatum. Facta carta apud Burgus II. ${ }^{a}$ die Marti era $M .{ }^{a} C C .{ }^{a} L X .{ }^{a}$. Et ego rex Fernando regnans in Castiella et 
Toleto hanc cartam quam fieri iussi manu propria roboro et confirmo. E yo sobredicho rey don Alfonso regnant en uno con la reyna donna Yolant mi mugier et con mis fijas la infante donna Berenguella e la infante donna Beatriz en Castiella en Toledo en Leon en Gallizia en Seuilla en Cordoua en Murcia en Jahen en Baeça en Badalloz e en el Algarue otorgo este priuilegio e confirmolo. Fecha la carta en Valladolit por mandato del rey. Tres dias andados del mes de setiembre en era de mille e dozientos e nonaenta e tres annos en el anno de don Odoart fijo primero e heredero del rey Henrric de Anglatierra recibio caballeria en Burgos del rey don Alfonso el sobredicho.

(1. ${ }^{a}$ col.) Don Sancho electo de Toledo cancller del rey confirma. Don Felippe electo de Seuilla confirma.

(2. ${ }^{a}$ col.) Don Alfonsso de Molina confirma. Don Frederich confirma. Don Henrrich confirma.

(3. ${ }^{a}$ col.) Don Alfonso fijo del rey Johan emperador de Constantinopla y de la emperadriz donna Berenguela conde Do e vasallo del rey confirma. Don Johan fijo del emperador e de la emperadriz sobredichos conde de Montfort uassallo del rey confirma. firma.

(4. ${ }^{a}$ col.) Don Johan arzobispo de Santiago chançeller del rey con-

$\left(5^{\circ}\right.$ col.) Don Manuel confirma. Don Ferrando confirma. Don Loys confirma.

Don Aboabdille Abennaçar rey de Granada vassallo del rey confirma. Don Mahomat Abemnahomat Abenhut rey de Murcia vassallo del rey confirma. Abemnathfot rey de Niebla vassallo del rey confirma.

(1. ${ }^{a}$ col.) Don Remondo obispo de Segouia confirma. Don Pedro obispo de Siguença confirma. don Gill obispo de Osma confirma. Don Mathe obispo de Cuenca confirma. Don benito obispo de Auila. Diago Lopez de Salzedo merino mayor de Castiella confirma. Garci Suarez merino mayor del Regno de Murcia confirma. Maestre Ferrando Notario del rey en Castiella confirma. Roy Lopez de Medoza almirage de la mar confirma. Sancho Martinez de Xodar adelantado de la frontera confirma. Garci Perez de Toledo Notario del rey en Andaluzia confirma. Gonçaluo 
Morant merino mayor de Leon confirma. Roy Suarez merino mayor de Gallizia confirma. Don Suero Perez electo de Çamora Notario del rey en Leon confirma. Johan Perez de Cuenca la escriuo en anno quarto que el rey don Alfonso regno. 
DOCUMENTO IV

1270, Agosto, 23. Burgos

Carta de compromiso entre Pérez Correa y Gutierre Suárez por el que la Ossa y Dos Barrios pasan a la Orden a cambio de otros territorios que Gutierre Suárez recibe.

AHN Ordenes Militares. Carp. 214, n. ${ }^{\circ} 18$

Conosçada cosa sea a quantos esta carta vieren como nos don Pelay Perez por la gracia de Dios Maestro de la cavalleria de la Orden de Santiago con otorgamiento de don Pedro Nuñez comendador mayor de Castilla e del Prior de Ucles e de todo el cabildo general de nuestra orden. E yo don Gutier Suarez por mi e por doña Elvira Cañes mi mogier e por toda mi nos façemos a tal pleito e a tal auenençia en que nos en razon de los emplazamientos que nos el maestre e nuestra Orden auiemos con don Suer Tellez hermano de vos don Gutier Suarez et doña Aldonça Ferrandez so mogier de todo aquello que ellos auian a devian auer en Palacios e en Amayuelas de fuso e en el Arroyo de Meneses en Lugar nombrado en Villa Senuierno e en Villauia que nos lexaron a so muertes. Estos lugares de susodicho quanto hy don Suer Tellez et doña Aldonça Ferrandez so mogier auian a so muerte e auer deuian que fincara en nos el Maestro e en la Orden sobredicha por rason del emplazamiento que con esto fizieran e damos a uos don Gutier Suarez para vos et para todos uuestros supcesores que los ayades livres et quitos por siempre jamas para uender para empeñar para enagenar e para fazer dello toda uuestra uoluntat assi en uida como en muerte. Et uos don Gutier Suarez e uestra mogier doña Elvira que uos partades et uos quitades de oy dia adelante de la villa que diezen Dos Varrios que tenedes de nos en tenençia por en uestros dias que nos uos auiemos dada 
por el lugar que dizen la Ossa que es un Mont Aragon que nos diestes por esta donaçion que nos fizieramos e que sigue en nos. $E$ yo don Gutier Suarez por mi e por mi mogier doña Elvira quanto menos de la villa sobre dicha que disen dos Barrios e de todos los derechos que hy abemos e deuemos auer por rason de la donacion que nos ende fisirades e donaremos nolla et lexamos nolla que la ayades liure e quita assi como la auiedes al tiempo que la dierades. Et dones a la Ossa que yaz en Mont aragon que la ayades nosa el maestre e nuestra Orden por juro de heredat por siempre iamas e que fagades ende toda nuestra uoluntad a todo timpo. Et que esto todo sea firme e en nuestra (borrado) en dubda fizimos desta auenencia fazer dos cartas paradas por Abeçe que la una que tengamos nos el maestre e nuestra Orden, e la otra que tenga yo don Gutier Suarez, seelladas de los seellos de nos el maestro e del cabildo de nuestra Orden e de los seellos de mi don Gutier Suarez e de Doña Elvira Yañes mi mogier. Fecha la carta en Vurgos veynte e tres dias de Agosto. Era mill Trezientos e ocho años. Testigos don Pedro Nuñez comendador mayor de Castiella, don Conçalo Royz Miguel Perez Johan Diez Pelay Rodriguez Freyres de la Orden sobredicha. Maestro Gomez. Ferran Yañes. Johan Uermudez alcaldes del Rey (borrado) Rodriguez cauallero de Çamora Pedro Suarez. Tel Garcia fijos de don Garcia Suarez. Garcia Perez cauallero de Villa Nueva e yo Marqo Perez escriuano del Rey fize estas cartas por mandado del maestro e de don Gutier Suarez sobredichos et so testigo. 

. 